\title{
A 'hijacked' regulatory mechanism
}

To disseminate between host cells, Listeria monocytogenes polymerizes host cell actin using ActA, a bacterial protein that mimics the action of Wiscott-Aldrich syndrome protein (WASP) and recruits the actinnucleating ARP2/3 complex to the bacterial surface. This results in the formation of actin tails, which are required for cell-to-cell migration. A study by Chong et al. now reveals that, in addition to mimicking the host cell protein, L. monocytogenes 'hijacks' the host cell mechanism that regulates this process.

In host cells, phosphorylation of the verprolin-cofilin-acidic (VCA) domain of WASP by the serine/threonine protein kinase CK2 (casein kinase 2) is required for its interaction with the ARP2/3 complex and subsequent actin polymerization. Preliminary screens identified CSNK2B (which encodes the regulatory $\beta$-subunit of $\mathrm{CK} 2$ ) as a candidate gene involved in L. monocytogenes cell-to-cell spread. On the basis of these data, the authors examined whether CK2 has a role in $L$. monocytogenes dissemination. They depleted CSNK2B from the host cells using small

L. monocytogenes has co-opted the mechanism by which the host cell regulates actin polymerization
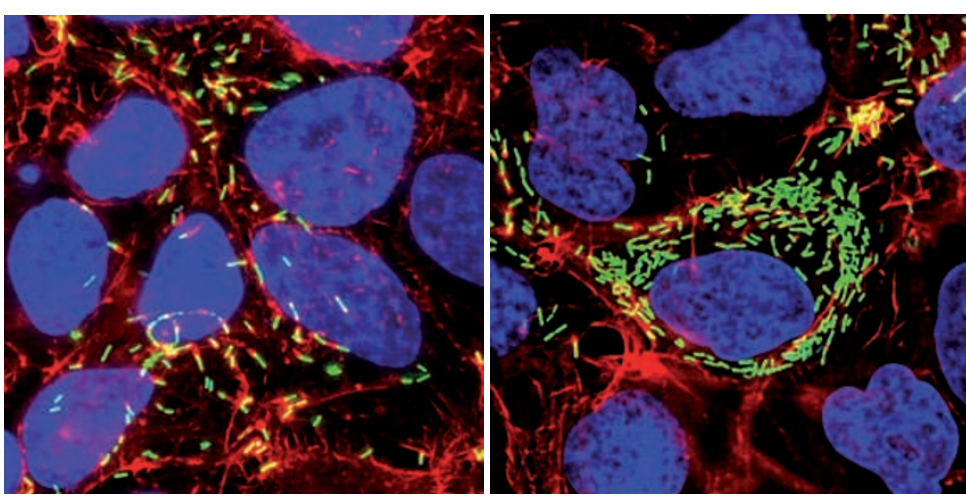

Control (left) and CSNK2B-depleted (right) cells infected with L. monocytogenes expressing green fluorescent protein and stained for filamentous actin (red); host cell nuclei are stained in blue. Bacteria infecting CSNK2B-depleted cells show defects in cell-to-cell spread and do not associate with actin. Image courtesy of R. Chong, Yale University School of Medicine, USA.

residues in this region decreased CK2-mediated phosphorylation. Furthermore, mutation of both serine residues resulted in reduced L. monocytogenes cell-to-cell spread and a defect in actin tail formation. This phenotype was partially rescued in bacteria with mutations that mimic phosphorylation, confirming that phosphorylation of these residues is necessary for actin tail formation.

So does CK2 phosphorylation of L. monocytogenes ActA mediate the interaction of ActA with the ARP2/3 complex, similarly to what is observed in host cells? In vitro assays showed that phosphorylation of ActA by CK2 markedly increased the interaction of ActA with the ARP2/3 complex. This depended on phosphorylation of the serine residues in the corresponding VCA domain of ActA by CK2, as their mutation abrogated ARP $2 / 3$ recruitment to the bacterial cell surface. Finally, mutation of these residues resulted in an attenuation of spleen colonization in an in vivo mouse model of L. monocytogenes infection, confirming the importance of Act A phosphorylation by CK2 in L. monocytogenes cell-to-cell spread.

Taken together, these findings show that phosphorylation of ActA by CK 2 mediates ActA binding to $\mathrm{ARP} 2 / 3$ and results in actin tail formation and consequent L. monocytogenes cell-to-cell spread. So, in addition to hijacking the structure of the VCA domain, L. monocytogenes has co-opted the mechanism by which the host cell regulates actin polymerization in a case of regulatory mimicry.

Rachel David

ORIGINAL RESEARCH PAPER Chong, R. et al. Regulatory mimicry in Listeria monocytogenes actin-based motility. Cell Host Microbe 6 , 268-278 (2009)

FURTHER READING Elde, N. C. \& Malik, H. S. The evolutionary conundrum of pathogen mimicry. Nature Rev. Microbiol. 7, 787-797 (2009). 